\title{
Opiniones y expectativas de pacientes con enfermedades relacionadas con la exposición al amianto
}

\section{Opinions and expectations of patients with health problems associated to asbestos exposure}

\author{
M.A. Prieto, A. Suess, J.C. March, A. Danet, O. Pérez Corral, A. Martín
}

\section{RESUMEN}

Fundamento. La prevalencia de enfermedades relacionadas con la exposición al amianto requiere el desarrollo de programas de vigilancia y protocolos de atención sanitaria específicas. El objetivo del estudio es conocer las opiniones y expectativas de los ex trabajadores de una fábrica de uralita, con el fin de adecuar el proceso asistencial a las necesidades de la población afectada, así como conocer la actividad de la asociación que les representa.

Métodos. Estudio cualitativo desarrollado con grupos focales con extrabajadores de una fábrica de uralita, miembros de la asociación AVIDA (Sevilla). Grabación y trascripción de las entrevistas. Análisis de discurso, con ayuda del programa Nudist Vivo 1.0.

Resultados. Todas las personas entrevistadas presentan problemas de salud, entre ellos asbestosis, cáncer de pulmón y mesotelioma. A través de la asociación, están en un proceso continuado de negociación con la administración pública para mejorar la atención sanitaria, lograr el reconocimiento como enfermedad laboral y el pago de indemnizaciones. Señalan como mayor problema del proceso asistencial actual la falta de seguimiento y continuidad en la atención. Valoran positivamente la creación de unidades de atención específicas, el trato humano recibido y la calidad de los instrumentos técnicos en el Sistema de Salud Público. En cambio, critican las dificultades de un diagnóstico acertado, la falta de continuidad asistencial, así como las dificultades burocráticas y la escasez de atención sanitaria específica a familiares afectados. Como expectativa de futuro, resaltan su interés en seguir participando en la elaboración de programas y protocolos.

Conclusiones. El estudio confirma el carácter multifactorial de las enfermedades relacionadas con la exposición al amianto, así como la relevancia de conocer las necesidades y demandas de la población afectada para mejorar la atención sanitaria específica.

Palabras clave. Amianto. Calidad asistencial. Participación de los pacientes. \begin{abstract}
Background. The prevalence of diseases related to asbestos exposure requires the development of monitoring programs and specific health care protocols. The aim of this study is to determine the opinions and expectations of former workers of an asbestos factory, in order to adapt the care process to the needs of the affected population, and to learn about the activity of the association that represents them.
\end{abstract}

Methods. Qualitative study. Focus groups with former employees of a corrugated asbestos factory, members of the association AVIDA (Seville). Recording and transcription of interviews. Discourse analysis with Nudist Vivo 1.0.

Results. All respondents have health problems, including asbestosis, lung cancer and mesothelioma. Through the association, they are involved in an ongoing process of negotiation with the public administration, to improve healthcare, achieve recognition as having an occupational disease and the payment of compensation. The lack of monitoring and continuity in care is designated as the major problem in the current care process. They welcome the creation of special care units, the good treatment received and the quality of technical instruments in the public health system. On the contrary, they criticize the difficulties in finding an accurate diagnosis, the lack of continuity of care, and the bureaucratic difficulties and lack of specific care directed to affected relatives. The participants' expectations highlight their intention to participate in the development of future programs and protocols.

Conclusions. This study confirms the multifactor nature of diseases related to asbestos exposure and the importance of determining the needs and demands of the affected population in order to improve health care.

Key words. Asbestos. Quality of health care. Patient.

An. Sist. Sanit. Navar. 2011; 34 (1): 33-42

Escuela Andaluza de Salud Pública. Granada. España

Recepción: 5 de octubre de 2010

Aceptación provisional: 19 de noviembre de 2010

Aceptación definitiva: 21 de enero de 2011

\section{Correspondencia:}

Alina Danet

Escuela Andaluza de Salud Pública.

Cuesta del Observatorio, 4

18011 Granada. España

E-mail: alina.danet.easp@juntadeandalucia.es 


\section{INTRODUCCIÓN}

A nivel mundial, se estima que hay alrededor de 2,3 millones de muertes por causas laborales al año ${ }^{1}$. En el ámbito español, en el año 2004 esta cifra se situó en $16.000^{2}$. Según un documento de la $\mathrm{OMS}^{3}$, alrededor de la mitad de las muertes por cáncer profesional se debe a la exposición al asbesto. La atención a las enfermedades relacionadas con la exposición al amianto constituye una línea estratégica prioritaria dentro de los programas de salud ocupacional y prevención de enfermedades profesionales, tanto a nivel internacional ${ }^{1,3,4}$, europeo $^{5}$, como en el ámbito español ${ }^{6-8}$.

El amianto o asbesto tiene múltiples aplicaciones en la construcción, la industria automovilística, ferroviaria, petroquímica, nuclear y naval ${ }^{3,6,9,10}$. Se puede distinguir entre diferentes tipos de amianto $^{4,6}$ y tipos de exposición ${ }^{3,6,9-12}$. Según la $\mathrm{OMS}^{3}$, la cifra de personas expuestas actualmente al amianto a nivel mundial es de 125 millones.

En la actualidad, existe un amplio consenso sobre las principales enfermedades relacionadas con una exposición prolongada al asbesto ${ }^{3,6,10,13-16}$, aunque sólo a principios del siglo XXI se inició la prohibición total de su producción, aplicación y comercialización ${ }^{17-19}$. En el ámbito de la Unión Europea, una directiva de $1999^{17}$ obliga a los estados miembros a la prohibición del uso de materiales que contienen asbesto, y otra directiva del 2003 la extracción, manufacturación y comercialización de amianto ${ }^{18}$. En España, la producción, manufacturación y comercialización se prohibió en el año $2001^{19}$.

Se han desarrollado recomendaciones y guías de actuación, entre ellas: la Declaración de Dresde sobre la Protección de los Trabajadores contra el Asbesto ${ }^{20}$, la guía de actuación desarrollada por la Agencia Europea para la Seguridad y la Salud en el Trabajo $^{21}$ o la guía de Helsinki ${ }^{22}$. Las primeras actuaciones en el ámbito español, datan de los años $60^{12}$, con un reconocimiento de la asbestosis como enfermedad profesional (1961), la introducción de exámenes médicos para trabajadores con exposición al asbesto (1963), la aprobación de un Reglamento sobre Trabajos con Riesgo de
Amianto y la prohibición del amianto azul $(1984)^{12}$. En 1999, se aprobó un Protocolo de Vigilancia Sanitaria Específica ${ }^{6}$, seguido por la creación de un Programa Integral de Vigilancia de la Salud de los Trabajadores $(2002)^{23}$ que han sido expuestos al amianto. En el año 2006, el Ministerio de Sanidad y Consumo publicó un Real Decreto ${ }^{24}$ que establece las disposiciones mínimas de seguridad y salud aplicables a los trabajos con riesgo de exposición al amianto.

En el ámbito del Servicio Andaluz de Salud, se creó en el año 2001 un grupo de trabajo intersectorial ${ }^{25}$, compuesto por organismos públicos y representantes de organizaciones sindicales y la Asociación de víctimas del amianto (AVIDA) ${ }^{26}$, que elaboró un protocolo de vigilancia y seguimiento y un estudio piloto con personas afectadas en la provincia de Sevilla, implantando el programa en tres hospitales de referencia ${ }^{25}$.

En la bibliografía reciente, se encuentran diferentes valoraciones de la relevancia de prevención, vigilancia y tratamiento de enfermedades relacionadas con la exposición de asbesto, tanto en trabajos publicados en el ámbito español ${ }^{6,9,10,12,23,26-28}$, como en el contexto internacional ${ }^{6,11,29-31}$.

En el año 2007 se inició, en el ámbito andaluz, la elaboración de un proceso asistencial para las enfermedades relacionadas con la exposición al amianto ${ }^{25}$. La Consejería de la Salud de Andalucía pidió a la Escuela Andaluza de Salud Pública un estudio sobre las opiniones y expectativas de las personas afectadas, con el fin de tener en cuenta sus necesidades específicas en el proceso de elaboración del proceso asistencial. El presente artículo ofrece un resumen de los aspectos más relevantes de este estudio, con un doble objetivo: conocer la actividad y experiencias de la asociación de víctimas del amianto (AVIDA) ${ }^{26}$ y recoger las opiniones, propuestas y expectativas de mejora de sus miembros respecto al proceso asistencial.

\section{MATERIAL Y MÉTODOS}

Se utilizó una metodología cualitativa, empleando la técnica del grupo focal. Los 
criterios de inclusión fueron: haber trabajado en un entorno laboral con exposición al amianto y tener problemas de salud relacionados con la exposición al mismo.

Para la selección de los participantes se contactó con AVIDA. Se trata de la única asociación existente en el ámbito andaluz de personas con enfermedades relacionadas con la exposición al amianto. Sus miembros son trabajadores posocupacionales de la fábrica de uralita Bellavista de Sevilla, cerrada en 1999, o familiares de los mismos. Se seleccionaron personas de diferentes edades y diferentes estados de salud, buscando la máxima variedad de experiencias y perspectivas respecto al objeto de estudio.

Para la recogida de datos se hicieron dos grupos focales en mayo de 2008.

Grupo focal 1, con los miembros de la Junta Directiva de la Asociación AVIDA. Los seis participantes eran hombres, trabajadores posocupacionales de la fábrica de uralita Bellavista, y afectados de enfermedades relacionadas con la exposición al amianto.

Grupo focal 2, con personas afectadas por enfermedades relacionadas con la exposición al amianto, 15 miembros de la asociación AVIDA, 14 hombres y una mujer. Los 14 hombres fueron trabajadores postocupacionales de la fábrica de uralita y afectados. La única mujer participante es hija de un difunto trabajador y abogada de AVIDA.

Se informó a los participantes sobre los objetivos de estudio, y se les presentó un documento de Consentimiento Informado por escrito, asegurándose la confidencialidad y el carácter anónimo de los datos personales.

En el grupo focal realizado con miembros de la Junta Directiva de la Asociación, se preguntó por la historia, los objetivos y actividades de la asociación.

Las preguntas del grupo focal se centraron en el estado de salud, la experiencia general con el sistema de salud público, el análisis de la situación actual de la atención en el sistema de salud público, y las propuestas de mejora. Se identificaron los puntos fuertes y débiles, así como las expectativas de mejora respecto a dos dimensiones: diferentes momentos en el proceso asistencial (primera cita en Atención Primaria, proceso de citación, primera cita en Atención Especializada, realización de pruebas, tratamiento, seguimiento) y diferentes dimensiones del modelo SERVQUAL ${ }^{32}$ (trato, cortesía, información, competencia, capacidad de respuesta, accesibilidad, adecuación de las instalaciones). (Tabla 1).

Las sesiones se grabaron y se transcribieron. Para el análisis de discursos, se utilizó el software NUDIST Vivo 1.0.

Tabla 1. Dimensiones de análisis.

\begin{tabular}{ll}
\hline \multicolumn{1}{c}{ Dimensiones } & \multicolumn{1}{c}{ Categorías } \\
\hline & - Atención Primaria \\
& - Primera cita \\
& - Derivación \\
Momentos del proceso & - Atención Especializada \\
asistencial & - Primera cita \\
& - Realización de pruebas \\
& - Tratamiento \\
& - Seguimiento \\
\hline & - Accesibilidad \\
& - Trato y cortesía \\
& - Información \\
Dimensiones del modelo & - Competencia \\
SERVQUAL & - Capacidad de respuesta \\
& - Tangibilidad \\
\hline
\end{tabular}




\section{RESULTADOS}

Los miembros de la asociación AVIDA muestran un alto nivel de conocimiento de la situación legislativa actual, así como un gran interés en participar en el proceso de mejora del proceso asistencial de enfermedades relacionadas con la exposición de amianto, haciendo hincapié en su experiencia prolongada de contacto y colaboración con la Administración Sanitaria Pública, tanto a nivel autonómico, como en el contexto estatal. Todos se muestran interesados en conocer los resultados de la auditoría y la continuación del proyecto de protocolización del proceso asistencial. Además, proponen que se entreviste también a trabajadores afectados de enfermedades relacionadas con la exposición al amianto que están todavía en activo, como los trabajadores de RENFE o de astilleros de Cádiz o Sevilla.

"Ojalá se hiciera esto que se ha hecho en Sevilla... pues se pudiera hacer con los astilleros de Cádiz, astilleros de Sevilla, RENFE, en Málaga... los compañeros de Córdoba, de Jerez”... (Afectado. Grupo focal).

En el segundo grupo focal realizado, todos los entrevistados señalan estar afectados por enfermedades relacionadas con la exposición al amianto, entre ellas asbestosis, cáncer de pulmón, mesotelioma pleural maligno, afectación de las vías respiratorias y sensibilidad aumentada a enfermedades comunes como la gripe o bronquitis. Se distinguen casos con un diagnóstico concreto y reconocimiento del problema de salud como enfermedad profesional, y afectados con una sintomatología difusa que ellos llaman "la antesala de la asbestosis".

También se señala la influencia de la enfermedad en el estado de ánimo, en consecuencia de la conciencia del carácter no tratable de la misma, y a causa de la experiencia repetida de acompañar el fallecimiento de ex-compañeros de trabajo. El riesgo para la salud de familiares y vecinos aparece como otra preocupación importante.

"Casi todos los meses tenemos que ir al cementerio a enterrar a un compañero". (Miembro de la asociación).

Respecto a la atención sanitaria recibida en el sistema sanitario público, parten de una evaluación negativa ("hay un abandono de la salud pública para con nosotros"), mejorándose la situación, según su opinión, con la introducción reciente de unidades clínicas especializadas en el ámbito andaluz. "Se observa una desigualdad en la calidad de atención entre afectados con un diagnóstico claro, y pacientes con sintomatología difusa o familiares afectados" (la abogada de la asociación e hija de un antiguo trabajador, así como tres personas más). Se señala una falta de homogeneidad en el abordaje sanitario de las enfermedades relacionadas con la exposición de amianto en diferentes comunidades autónomas.

"El sistema sanitario tiene un comportamiento muy desigual a nivel del Estado, (...) no hay una situación homogénea de conocimiento del tema”. (Miembro de la Junta Directiva).

Sobre la evaluación del proceso asistencial (Tabla 2), en Atención Primaria, como principal dificultad se nombra la frecuente falta de conocimientos específicos por parte de los médicos de familia, así como la falta de coordinación con Atención Especializada. Como otro punto de crítica se nombra la falta de interés por la situación de los familiares afectados.

"Los médicos de cabecera en una inmensa mayoría no tienen ni idea de lo que es una asbestosis". (Miembro de la asociación).

A nivel de Atención Especializada, se constata la frecuencia de una falta de sospecha clínica, o de diagnósticos erróneos, especialmente a causa de una lectura deficiente de las radiografías. 
Tabla 2. Fortalezas y debilidades en diferentes momentos del proceso asistencial.

\begin{tabular}{|c|c|c|c|}
\hline Nivel asistencial & Proceso asistencial & Fortalezas & Debilidades \\
\hline \multirow[t]{2}{*}{ Atención Primaria } & $\begin{array}{l}\text { - Primera cita } \\
\text { - Seguimiento }\end{array}$ & & $\begin{array}{l}\text { - Falta de conocimientos } \\
\text { específicos del médico de familia } \\
\text { - Falta de atención a los familiares }\end{array}$ \\
\hline & - Derivación & & - Falta de coordinación \\
\hline \multirow{2}{*}{$\begin{array}{l}\text { Atención } \\
\text { Especializada }\end{array}$} & $\begin{array}{l}\text { - Primera cita } \\
\text { - Realización de pruebas } \\
\text { - Diagnóstico } \\
\text { - Tratamiento }\end{array}$ & $\begin{array}{l}\text { - Trato recibido } \\
\text { - Participación en los } \\
\text { procesos de decisión } \\
\text { - Medios técnicos } \\
\text { - Atención a las familias }\end{array}$ & $\begin{array}{l}\text { - Falta de sospecha clínica } \\
\text { - Periodicidad insuficiente de las } \\
\text { pruebas } \\
\text { - Frecuencia de diagnósticos } \\
\text { erróneos } \\
\text { - Falta de recursos humanos }\end{array}$ \\
\hline & - Seguimiento & & $\begin{array}{l}\text { - Falta de continuidad asistencial } \\
\text { - Cambio frecuente de médicos } \\
\text { - Dificultades administrativas } \\
\text { - Falta de cumplimiento de los } \\
\text { protocolos } \\
\text { - Proceso de citación en la cita } \\
\text { anual }\end{array}$ \\
\hline $\begin{array}{l}\text { Aspectos } \\
\text { generales }\end{array}$ & & $\begin{array}{l}\text { - Mejora del proceso } \\
\text { a través de la } \\
\text { introducción de } \\
\text { unidades clínicas } \\
\text { especializadas }\end{array}$ & $\begin{array}{l}\text { - Desigualdad en la calidad de } \\
\text { atención según el lugar de } \\
\text { residencia y la sintomatología } \\
\text { - Falta de un protocolo unificado } \\
\text { - Falta de atención a aspectos } \\
\text { sociales de la enfermedad }\end{array}$ \\
\hline
\end{tabular}

En cambio, se evalúa positivamente el trato recibido por parte de los profesionales sanitarios, la posibilidad de participación en los procesos de toma de decisión sobre pruebas y tratamientos, la calidad de los medios técnicos (máquinas de pruebas) y la atención sanitaria recibida en las tres unidades clínicas específicas existentes en el territorio andaluz, así como la inclusión de familiares en los protocolos de atención. Como propuestas de mejora (Tabla 3), se demanda la realización de actividades de formación continua dirigidas a médicos especialistas, así como el aumento de periodicidad de las pruebas. En relación a las unidades clínicas específicas, se propone la introducción de la figura de un especialista en Oncología y la implementación de unidades en otras zonas con población expuesta al amianto.

"Nuestro objetivo ha sido poner en marcha algo que nunca se había puesto y era el constituir las unidades médicas específicas (...) para atender la situación post-ocupacional de todos los trabajadores del amianto". (Miembro de la Junta Directiva)

Es en la fase de seguimiento donde se centran las críticas más agudas sobre el proceso asistencial. Se denota una falta de continuidad asistencial debida a cambios frecuentes de médicos, y recorridos administrativos complicados, así como una falta de cumplimiento de los protocolos establecidos. Todos los participantes refieren estos problemas.

"Nosotros en líneas generales venimos a decir que los médicos sean coherentes con el protocolo fijado por el consejo interterritorial". (Miembro de la Junta Directiva)

Como propuestas de mejora (Tabla 3) se nombran la introducción de una tarjeta para apuntar las citas periódicas, la creación de 
una base de datos informatizada, el envío de una carta recordatoria, un aumento de fre- cuencia de las citaciones, así como la garantía de atención por un mismo profesional.

Tabla 3. Propuestas de mejora que ofrecen los entrevistados.

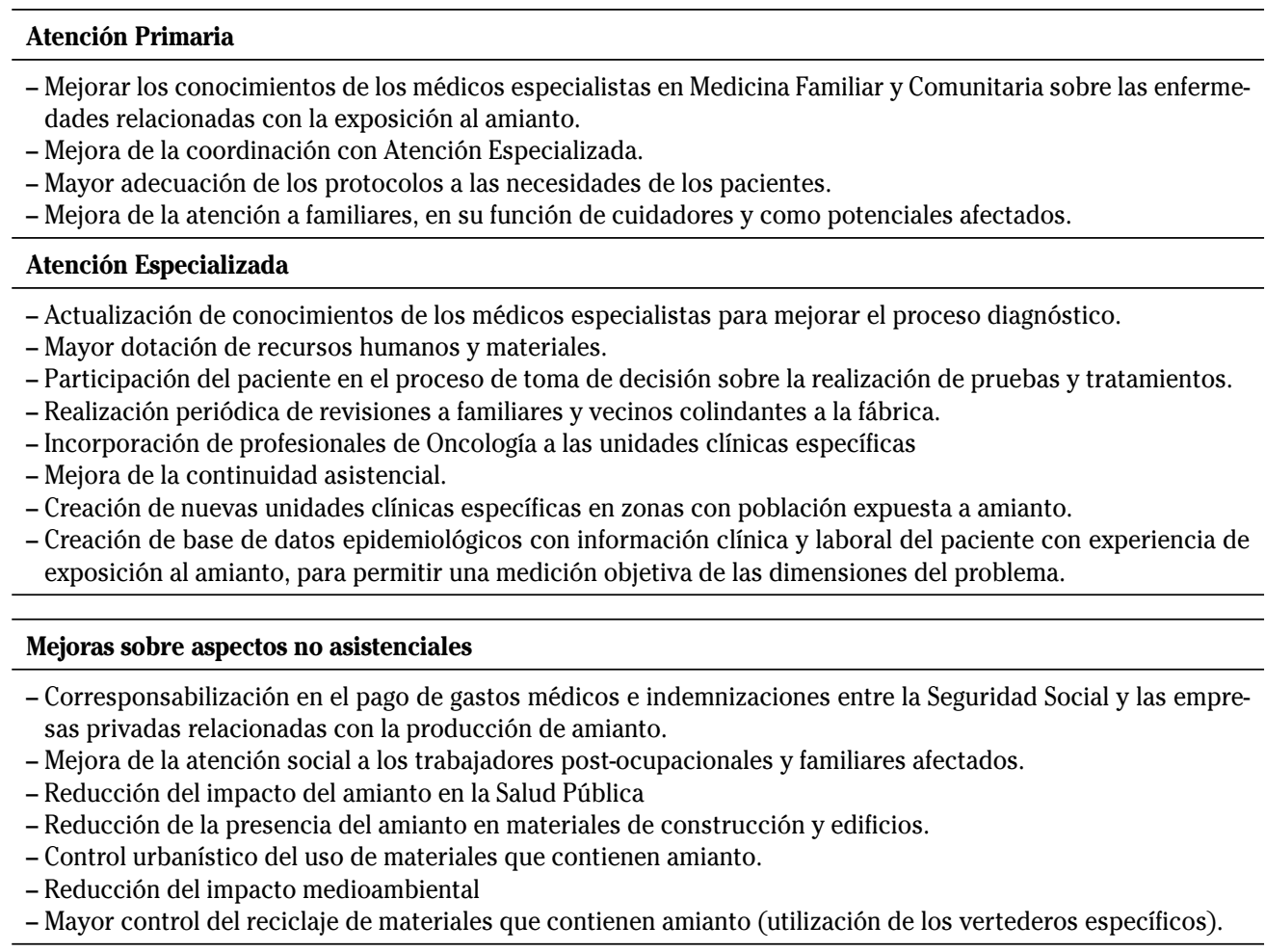

"Nosotros sugerimos algo tan elemental, tan básico, tan de forma continuada que funciona en las distintas especialidades médicas que es la típica tarjetica en la que te aconsejan cuando es la próxima revisión". (Miembro de la Junta Directiva).

Las personas entrevistadas realizan también reivindicaciones de carácter social, laboral y medioambiental, destacando el carácter multidimensional del problema. Proponen una corresponsabilidad entre la Administración Pública y las empresas privadas acerca del proceso de indemnización, señalan la necesidad de una mejora de la atención social a las necesidades de pacientes posocupacionales, familiares $\mathrm{y}$ vecindario afectado, y demandan un proceso de reducción del amianto en el entorno medioambiental. Contra una evaluación del problema como limitado a los trabajadores afectados, destacan la dimensión de salud pública presente en el abordaje de las consecuencias del amianto.

"Porque lo que era en un origen un problema de salud laboral, se ha convertido en un problema de salud pública, esa es la dimensión que tiene el problema”. (Miembro de la Junta Directiva).

\section{DISCUSIÓN}

La técnica de grupo focal ha dado respuesta a los objetivos del estudio, ya que se recomienda en la bibliografía especializada para conocer en profundidad las 
opiniones de un grupo de población, sus vivencias, sentimientos, necesidades $\mathrm{y}$ expectativas $^{33}$. La metodología grupal permite identificar el proceso de elaboración de los discursos, sus matices y contradicciones internas ${ }^{34}$. La metodología elegida no posibilita la generalización de los resultados, sin embargo, esta limitación no anula la representatividad y validez de los discursos recogidos. Las conclusiones que se generan del estudio tienen la limitación de no ser extrapolables, pero sí describen y explican una realidad concreta que puede encontrarse en otros contextos sociolaborales similares.

La evaluación del amianto como "un problema de salud pública" por parte de los miembros de la Junta Directiva de la asociación AVIDA coincide con el análisis realizado por Cárcoba $^{27}$ que utiliza esta misma expresión para caracterizar el problema de la exposición al amianto, además de constatar -en un artículo publicado en 2001- una falta de debate social y prohibición legal (aprobada en el ámbito español a finales de $2001^{22}$ ). En un texto posterior, Cárcoba ${ }^{28}$ plantea la pregunta por el "amianto después del amianto", destacando que con la prohibición de la manufacturación y comercialización no desaparece el problema de salud, por el alto grado de contaminación aún presente. Las propuestas que coinciden con algunas de las demandas de los miembros de la asociación AVIDA, entre otros el apoyo a las víctimas, la creación de un registro nacional de afectados, la realización de estudios epidemiológicos, el establecimiento de un fondo de indemnización, el reconocimiento como enfermedad profesional, la eliminación de la exposición pasiva, la creación de un inventario de los edificios afectados y la introducción de un proceso regulado de retirada.

Las personas entrevistadas del presente estudio hablan de las dimensiones no sólo físicas, sino también emocionales del problema. Resaltan la vivencia reiterada de pérdida de compañeros de trabajo, una sensación de impotencia frente al carácter incurable de las enfermedades que padecen, así como la conciencia de haber sido expuestos a un entorno laboral cuyos riesgos se conocían desde hace tiempo. La discrepancia entre el conocimiento físico y la regulación jurídica restrictiva en la última mitad del siglo XX lleva a algunos autores $^{12,29}$ a hablar de las "tragedias de las sociedades industriales"12, de una "historia de ocultación"12 del saber experto, levantando la pregunta "if preventable, why not prevented" (si prevenible, por qué no prevenida") $)^{29}$.

Los miembros de la asociación AVIDA ofrecen la imagen de un grupo activo en contacto continuado, que contrasta con los ex-trabajadores de uralita incluidos en otro estudio ${ }^{27}$, que sólo se encuentran de nuevo cuando ya están en el hospital. En contra de una imagen social del activismo asociativo limitado a una actitud de queja y protesta, los entrevistados dan una valoración matizada de su situación, en la que señalan los puntos que consideran deficientes, a la vez de valorar los aspectos de mejora logrados en los últimos años. En este sentido, su presentación pública coincide con la valoración de las asociaciones como nuevo actor social, altamente informado e interesado en una participación activa en la gestión sanitaria, tal como se propone en la bibliografía reciente $\mathrm{e}^{35,36}$.

En coherencia con García y col $^{9}$, las personas entrevistadas del presente estudio confirman que el Protocolo de Vigilancia Sanitaria aprobado en el 2002 que establece como objetivo la creación de un Registro de personas afectadas, sigue sin cumplirse en su totalidad. También otros autores ${ }^{10}$ resaltan la importancia de un registro de afectados, proponiendo su análisis por cohortes de edad, así como la obligación ética de reconocimiento del origen laboral del problema.

Pascal y Marín ${ }^{23}$ describen los reconocimientos médicos periódicos, tanto al principio de la actividad profesional con riesgo de exposición, durante el periodo laboral y en la fase postocupacional establecidos por el Protocolo de Vigilancia Sanitaria Específica. Los miembros de la asociación AVIDA señalan la relevancia de una mayor homogeneización y accesibilidad de los 
procesos de revisión, estimando que el establecimiento de un proceso asistencial puede ayudar a una mejora del seguimiento que en la actualidad evalúan como deficiente.

Además de la necesidad de una atención sanitaria específica, los entrevistados señalan el carácter más amplio de la herencia del amianto, en el sentido de una contaminación medioambiental continuada que requiere actuaciones políticas no limitadas al sector sanitario, coincidiendo en esta valoración con otros autores ${ }^{12,27,28,30}$.

El asbesto sigue planteando retos a la salud ambiental y laboral, considerándose en el contexto estudiado como un problema de salud pública que requiere la elaboración de protocolos específicos de atención sanitaria a la población afectada. La asociación de ex-trabajadores de amianto presenta un alto grado de información y voluntad de participar en la elaboración de programas de vigilancia y seguimiento de las enfermedades relacionadas con el amianto. Conocer sus opiniones y demandas se revela como un requisito previo relevante para el desarrollo de una atención sanitaria que pueda dar respuesta a las necesidades específicas de este grupo de población.

\section{BIBLIOGRAFÍA}

1. OMS, Organización Mundial de Salud. Seoul Declaration on Safety and Health at Work. http://www.issa.int/aiss/content/download/43103/824949/file/2Seoul_Declaration. pdf (consultada 01/02/2010).

2. García AM, GADEA R, LóPEz V. Estimación de la mortalidad atribuible a enfermedades laborales en España. Rev Esp Salud Pública 2007; 81: 261-270.

3. OMS, Organización Mundial de Salud. Eliminación de las enfermedades relacionadas con el amianto. http://whqlibdoc.who. int/hq/2006/WHO_SDE_OEH_06.03_spa.pdf (consultada 01/02/2010).

4. OMS, Organización Mundial de Salud. Outline for the Development for National Programs for Elimination of Asbestos-Related Diseases. http://whqlibdoc.who.int/hq/2007/ WHO_SDE_PHE_07.02_eng.pdf (consultada 01/02/2010).
5. Agencia Europea para la Seguridad y la Salud en el Trabajo. SLIC European Asbestos Campaign. http://osha.europa.eu/campaigns/asbestos (citada 01/02/2010).

6. Comisión de Salud Pública. Consejo Interterritorial del Sistema Nacional de Salud. Protocolos de Vigilancia Sanitaria Específica. Amianto. Madrid: Ministerio de Sanidad 1999. http://www.msc.es/ciudadanos/saludAmbLaboral/docs/amianto.pdf (consultada 01/02/2010).

7. Ministerio de Sanidad y Consumo. Programa Integral de Vigilancia de la Salud de los trabajadores que han estado expuestos a amianto. Madrid: Ministerio de Sanidad y Consumo, 2004. www.msc.es/ciudadanos/ saludAmbLaboral/docs/protocoloAmianto. pdf (consultada 01/02/2010).

8. Servicio Andaluz de Salud. Seguimiento $\mathrm{y}$ atención a personas en contacto en el amianto. http://www.juntadeandalucia.es/ servicioandaluzdesalud/contenidos/publicaciones/datos/241/html/AtencionPersonasAmianto.pdf (consultada 01/02/2010).

9. García M, Artieda L, Esteban V, Guzmán A, CamiNo F, MARTínEz A et al. La vigilancia de la salud de los trabajadores expuestos al amianto: ejemplo de colaboración entre el sistema de prevención de riesgos laborales y el Sistema Nacional de Salud. Rev Esp Salud Pública 2006; 50: 27-59.

10. Aвú-Shams K, Pascal I. Características, propiedades, patogenia y fuentes de exposición del asbesto. An Sist Sanit Navar 2005; 28 (Supl.I): 7-11.

11. CAgLE PT. Criteria for attributing lung cancer to asbestos exposure. Am J Clin Pathol 2002; 117: 9-15.

12. MenÉndez A. Alice - A fight for life (1982) y la percepción pública de los riesgos laborales del amianto. Rev Med Cine 3; 2007: 49-56.

13. Luis G, Hernández C, Rubio C, Frías I, gutiérrez A, HARDISSON A. Toxicología del asbesto. Cuad Med Forense 2009, 57: 207-213.

14. Harding A-H, Darton A, Wegerdt J, McElenny D. Mortality among British asbestos workers undergoing regular medical examinations (1971-2005). Occup Environ Med 2009: 66: 487-495.

15. Lin R-T, TaKashashi K, Karjalainen a, Hoshuyama T, WiLson D, KAMEDA T et al. Ecological association between asbestos-related diseases and historical asbestos consumption: an historical analysis. Lancet 2007; 369: 844-849. 
16. Reid A, Heyworth J, KLerk de N, Musk AW. The mortality of women exposed environmentally and domestically to blue asbestos at Wittenoom. Western Australia. Occup Environ Med 2008; 65: 743-749.

17. Agencia Europea para la Seguridad y la Salud en el Trabajo. Directrice 1999/77/EC http:// osha.europa.eu/campaigns/asbestos/legislation.htm (consultada 01/02/2010).

18. Agencia Europea para la Seguridad y la Salud en el Trabajo. Directrice 2003/18/EC http:// osha.europa.eu/campaigns/asbestos/legislation.htm (consultada 27/02/2008).

19. Ministerio de Presidencia. Orden de $7 \mathrm{de}$ diciembre de 2001. Limitaciones a la comercialización y al uso de ciertas sustancias y preparados peligrosos. BOE 299; 14 de diciembre de 2001. http://www.boe.es/ boe/dias/2001/12/14/pdfs/A47156-47157.pdf (consultada 01/02/2010).

20. European Conference, Dresden Declaration on the Protection of Workers against Asbestos. http://www.hvbg.de/d/asbest/erklaer/ declarat.pdf (consultada 26/02/2010).

21. Agencia Europea para la Seguridad y la Salud en el Trabajo. SLIC European Asbestos Campaign. http://osha.europa.eu/campaigns/asbestos (consultada 26/02/2008).

22. Consensus Report. Asbestos, asbestosis and cancer: the Helsinki criteria for diagnosis and attribution. Scand J Work Environ Health 1997; 23: 311-316.

23. Pascal I, Marín Martínez B. Trabajadores en contacto con amianto: pautas de seguimiento. An Sist Sanit Navar 2005; 23 (Supl.1): 4549.

24. Ministerio de Trabajo y Seguridad Social. Orden del 31 de octubre de 1984. Reglamento sobre Trabajos con Riesgo de Amianto. BOE 267; 7 de noviembre de 1984. http://www. boe.es/boe/dias/1984/11/07/pdfs/A3214532149.pdf (consultada 01/02/2010).

25. Servicio Andaluz de Salud. Seguimiento y atención a personas en contacto en el amianto. http://www.juntadeandalucia.es/servicioandaluzdesalud/contenidos/publicaciones/datos/241/html/AtencionPersonasAmianto.pdf (consultada 27/02/2008).

26. Asociación de Víctimas del Asbesto AVIDA. www.avida.es (consultada 25/02/2008).

27. CÁRCoBA AC. El amianto en España: situación actual y perspectivas. Arch Prev Riesgos Labor 2001; 4: 58-60.

28. CÁrcoba AC. El amianto después del amianto. http://www.asepal.es/adjuntos/fichero_ 530_20070314.pdf (consultada 01/02/2010).

29. Greenberg M. Asbestos: medical and legal aspects. Int J Occup Environ Health 2006; 12: 87.

30. Marier M, Charney W, Rousseau R, Lanthier R, VAN RAALTE J. Exploratory sampling of asbestos in residences near thetford mines: the public health threat in Quebec. Int J Occup Environ Health 2007; 13: 386-393.

31. Amezcua M. La entrevista en grupo. Características, tipos y utilidades en investigación cualitativa. Enf Clínica 2003; 13: 112-117.

32. Parasuraman A, Zeithaml VA, Berry Ll. SERVQUAL: A multiple item scale for measuring consumer perceptions of quality service. J Retail 1988; 64: 12-40.

33. March Cerdá JC, Prieto Rodríguez MA, Hernán García M, Solas Gaspar O. Técnicas cualitativas para la investigación en salud pública y gestión de servicios de la salud: algo más que otro tipo de técnicas. Gac Sanit 1999, 13: 312-319.

34. Costa-Font, J. Participación colectiva y revelación de preferencias sobre programas sanitarios: un enfoque de sistema sanitario. Gac Sanit 2005; 19: 242-252.

35. Ringo WP. Asbestos as a work hazard: determining what was known and when. Professional Safety 2004; 49: 51-56.

36. Meneu R. Participación de los pacientes en las decisiones sobre su asistencia sanitaria. Rev Calidad Asistencial 2005; 20: 337-342. 